Sharif University of Technology
Scientia Iranica
Transactions E: Industrial Engineering
http://scientiairanica.sharif.edu

\title{
A joint scheduling of production and distribution operations in a flow shop manufacturing system
}

\author{
S. Mohammadi, A. Cheraghalikhani, and R. Ramezanian* \\ Department of Industrial Engineering, K.N. Toosi University of Technology, Tehran, Iran.
}

Received 29 May 2016; received in revised form 3 October 2016; accepted 18 December 2016

\author{
KEYWORDS \\ Integrated modeling \\ approach; \\ Flow shop scheduling; \\ distribution with \\ routing; \\ Imperialist \\ competitive \\ algorithm.
}

\begin{abstract}
In traditional scheduling problems and many real-world applications, the production operations are scheduled regardless of distribution decisions. Indeed, the completion time of a job in such problems is defined traditionally as the time when the production sequences of a job are finished. However, in many practical environments, completed orders are delivered to customers immediately after production stages without any further inventory storage. Therefore, in this paper, we investigate an integrated scheduling model of production and distribution problems simultaneously. It is assumed that products proceed through a permutation flow shop scheduling manufacturing system and are delivered to customers via available vehicles. The objective of our integrated model is to minimize the Maximum Returning Time (MRT), which is the time it takes for the last vehicle to deliver the last order to a relevant customer and return to production center. The problem is formulated mathematically, and then an Improved Imperialist Competitive Algorithm (I-ICA) is proposed for solving it. Furthermore, a sufficient number of test problems are generated for computational study. Various parameters of the algorithm are analyzed to calibrate the algorithm by means of the Taguchi method. At the end, the effectiveness of the proposed model and suggested algorithm is evaluated through a computational study where the obtained results show the appropriate performance of the integrated model and solving approach in comparison to those of the other algorithms.
\end{abstract}

(C) 2018 Sharif University of Technology. All rights reserved.

\section{Introduction}

Due to market globalization, emphasizing the coordination and integration among various functions of a supply chain has become one of the vital strategies of modern manufacturers to gain competitive advantages. The supply chain management has become one of the most important and widely discussed topics in man-

\footnotetext{
*. Corresponding author. Tel.: +982184063365;

Fax: +982188674858

E-mail addresses: mohammadi@kntu.ac.ir (S.

Mohammadi); Ali_Cheraghalikhani@mail.kntu.ac.ir (A.

Cheraghalikhani); Ramezanian@kntu.ac.ir (R. Ramezanian)
}

doi: $10.24200 /$ sci. 2017.4437 ufacturing research area over the last years [1]. Most of traditional scheduling literature reviews consider job sequencing regardless of the delivery considerations or by assuming delivery after production. Nevertheless, it is no longer suitable, and so the models should be extended with transportation considerations to cope with the distribution issue after production. There are only a few researchers that have considered the joint optimization of machine scheduling and job delivery in the integrated production and delivery scheduling; moreover, after a manufacturing company receives orders from customers, the company processes these orders on the machines, and then delivers the finished orders to the customers. This means that the finished products are delivered immediately to the relevant cus- 
tomers. Considering the importance and application of flow shop systems in production systems on one the hand and the significance of transportation issues, especially vehicle routing decisions on the other hand, the aim of this paper is the employment of integrated approach to production and distribution in a flow-shop manufacturing system.

In this paper, a new integrated model is developed for a flow shop and delivery-scheduling problem. In the proposed model, a Permutation Flow Shop Scheduling Problem (PFSSP) is assumed for production system. Indeed, in the integrated model, not only starting and finishing times of each job are determined on each machine, but also route of vehicles is determined for achieving optimum MRT through both production and delivery stages simultaneously. In addition, Improved meta-heuristic, Imperialist Competitive Algorithm (IICA) was proposed to solve the constructed model. To improve the performance of ICA, some effective policies are added to explore and exploit the solution space in assimilation and revolution steps of ICA and the resulting algorithm, called I-ICA, in this paper. To evaluate the performance of developed model and algorithm, we used three well-known datasets of flow shop scheduling problem in literature to generate suitable test problems. Next, to enhance the performance of the ICA, the algorithm parameters are adapted by preexperimental planning. On the other side, in order to investigate the value of integration by the developed model, three benchmarks are used as the data for production scheduling part of integrated model, and the distribution with routing data is generated randomly for each of production benchmarks. In order to evaluate the value of integration by the developed model, MRT is used as a performance measure which is defined as the time it takes for a vehicle to deliver the last order to a relevant customer and return to production center. Based on MRT comparison, effectiveness of the developed model is investigated where the obtained results show the adequate performance of the integrated model. At the end, the statistical analysis results show acceptable performance of the proposed model rather than non-integrated approach. Indeed, we evaluate the value of integration by comparing the proposed integrated approach with the hierarchical approach.

The reminder of the paper is structured as follows. Section 2 introduces the problem and develops a mathematical model. Extensive study of the related literature review is done in Section 3. Section 4 presents an improved solution algorithm based on ICA. The computational study and simulation results are provided in Section 5. Managerial insight, which includes examples of real-world application of the proposed model and its sensitivity analysis, is presented in Section 6. Section 7 contains the conclusions and some directions for future research.

\section{Literature review}

The integrated production and delivery scheduling was first studied by Potts [2]. Potts [2] and Hall and Shmoys [3] studied a single-machine scheduling problem with non-identical delivery and release times, where there are enough number of vehicles to immediately deliver products to a customer. Afterwards, Matsuo [4] considered a similar problem in the singlemachine environment in order to couple production with delivery decisions. Herrmann and Lee [5], Yuan [6], Chen [7], and Cheng et al. [8] evaluated that products are delivered after being processed by infinite delivery capacity via enough number of vehicles. Yang [9] and Hall et al. [10] considered some additional assumptions of such a problem. Yang [9] proposed a model similar to the one studied by Cheng et al. [8], yet with given delivery dates. Hall and Potts [11] suggested a variety of scheduling and delivery problems within a supply chain and minimized the overall scheduling and delivery costs. Transportation capacity is not considered in their model. Moreover, they assumed that each vehicle would be shipped to only one downstream destination. Lee and Chen [12] investigated other machine scheduling models by imposing constraints on both transportation capacity and transportation time. Hall and Potts [13] studied a variety of single-machine scheduling problems with the objective of minimizing the overall scheduling and delivery costs. One of the notable characteristics in their model is that there are always transporters available for delivering. Chang and Lee [1] further studied this problem where each job requires different physical spaces for delivery. Garcia et al. [14] dealt with several plants, each equipped with parallel machines and a fleet of vehicles that can deliver no more than one job at a time. Li et al. [15] developed a single-machine scheduling model that incorporates routing decisions of a delivery vehicle, which serves customers at different locations. The objective is to minimize the sum of job arrival times. Meanwhile, they developed a polynomial time algorithm for the case when the number of customers is fixed. He et al. [16], Zhong et al. [17], and Su et al. [18] revisited the problem of Chang and Lee [1] and improved algorithms. Li and Vairaktarakis [19] focused on a similar problem with jobs that consist of two jobs. There is a special machine for each job. Wang and Cheng [20] introduced the machine availability constraint into Lee and Chens' [12] model. Zhong et al. [17] presented some improved approximation results for the problems considered by Chang and Lee [1]. Chen and Lee [21] considered a more general two-stage scheduling problem where, in the second stage of transportation, there are multiple (more than two) transportation modes to select multiple destinations. Lu et al. [22] considered the singlemachine scheduling with release dates and job delivery. 
Liu and Lu [23] provided an improved approximation algorithm for the problem studied by Lu et al. [22].

Woeginger [24] considered a scheduling problem on parallel machines without considering release dates and proposed a heuristic method with worst-case analysis. Wang and Cheng [25] studied a parallel machine scheduling to integrate scheduling with delivery. In another study, Hall and Potts [26] analyzed the complexity of scheduling problems for many different objectives on single and parallel machine(s) with delivery coordination. Chen and Vairaktarakis [27] extended the problem to more destinations and an infinite number of vehicles with a threshold on loading capacity limited to a maximum number of jobs. Single as well as parallel machine scheduling problems are addressed in their research. In study of $\mathrm{Su}$ et al. [18], jobs are processed by two parallel machines and delivered to a customer with the objective of minimizing the makespan. Ullrich [28] integrated the production and outbound distribution scheduling in order to minimize total tardiness. The overall problem consists of two sub problems. The first addresses scheduling of a set of jobs on parallel machines with machine-dependent ready times, while the second focuses on the delivery of completed jobs with a fleet of vehicles with different loading capacities and ready times. Job-dependent processing times, delivery time windows, service times, and destinations were also taken into account in a study done by Ullrich [28]. Wan and Zhang [29] investigated a coordinated scheduling problem on parallel identical machines with job delivery. The objective is to minimize the sum of job arrival times. Moreover, Lee [30] proposed a coordinated scheduling of production and delivery issues, where jobs are processed on a single machine without preemption and then delivered to a customer. It was assumed that the delivery cost depends on time period for delivery, but not dependent on individual jobs. Lu et al. [31] integrated production and delivery scheduling on a serial batch machine to minimize the makespan. Two-machine flow shop scheduling with transportation constraints was suggested by Yuan et al. [32] to minimize makespan. Pan et al. [33] focused on a class of two-machine flow shop problem in which jobs need to be delivered to customers by vehicles after production stages. Finally, it should be noted that the complexity of integrated models with different assumptions was studied by many researchers in literature (e.g. $[1,15,29,30,34])$.

As can be seen, all the reviewed studies have just investigated the concept of integration of production and delivery of finished products to customers in singlemachine, parallel machine(s), and two-machine flow shop environments. To the best of our knowledge, there is no research in literature proposing $M$ machines permutation flow shop in integrated production and routing delivery model with vehicles. It seems that the high difficulty of such an assumption is one of the main reasons, while PFSSP is a well-known class of scheduling problem with many applications in industrial and manufacturing systems rather than single and parallel machines that have been extensively studied within recent years.

\section{Problem formulation}

In this work, we developed an integration model of the PFSSP and distribution with routing. In the developed model, not only starting and completion times of orders in each production machines should be calculated, but also the time of each vehicle departing from center, the rout of each vehicle and time of receiving orders to relevant customers, and total completion time for each order are considered as decision variables. Therefore, it seems that the proposed model is more complicated than traditional PFSSP and belongs to the class of NPhard problems.

\section{Problem parameters:}

$N \quad$ Number of jobs (customers order)

$M \quad$ Number of machines

$V \quad$ Number of vehicles

$J_{i} \quad$ Job number $i$

$O_{i m} \quad m$ th operation of $J_{i}$

$U \quad$ Very large positive number

$p_{i m} \quad$ Processing time of $O_{i m}$

$f_{i j v} \quad$ Travel time between customers $i$ and $j$ by vehicle $v$

$q_{i} \quad$ Units of vehicles capacity needed to carry $J_{i}$ that is identical for all vehicles

$Q_{v} \quad$ Capacity of vehicle $v$

$s_{j} \quad$ Service time at the $J$ th customer

Decision variables:

$t_{i m} \quad$ Starting time of $O_{i m}$

$Z_{i j} \quad$ A binary variable that indicates if $J_{i}$ precedes $J_{j}$

$y_{i v} \quad$ A binary variable that indicates if job $J_{i}$ is delivered by vehicle $v$

$x_{i j v} \quad$ A binary variable that indicates if arc $(i, j)$ is part of a vehicle route $v$

$b_{i v} \quad$ Starting time of service to customer $i$

in customer location

$S T_{v} \quad$ Starting time of vehicle $V$ route

$y y_{i j v} \quad$ Auxiliary variable that is equal to $y_{i v} * y_{j v}$

$t_{\max } \quad$ Time when final vehicle returns to production center

By the above notations, the proposed model is as follows (It should be noted that $i=0$ or $j=0$ in the 
proposed model's formulation refers to shop center):

$$
\text { Minimise MRT }
$$

Subject to $(1-14)$ :

$$
\begin{aligned}
& t_{i m}+p_{i m} \leq t_{j m}+U\left(1-Z_{i j}\right) t_{j m}+p_{j m} \leq t_{i m}+U Z_{i j} \\
& j \neq j, \quad i, j=1,2, \ldots, N, \quad m=1,2, \ldots, M, \\
& t_{i m}+p_{i m} \leq t_{i(m+1)} \\
& i=1,2, \ldots, N, \quad m=1,2, \ldots, M-1, \\
& \sum_{\nu=1}^{V} y_{i \nu}=1, \quad i=1,2, \ldots, N,
\end{aligned}
$$$$
\sum_{j=1}^{N} x_{0 j \nu}=1, \quad \nu=1,2, \ldots, V
$$$$
y_{i \nu} \leq \sum_{j=0}^{N} x_{j i \nu}, \quad i=1,2, \ldots, N, \quad \nu=1,2, \ldots, V,
$$$$
x_{i j \nu} \leq y_{i \nu} * y_{j \nu}
$$$$
i \neq j, \quad i, j=1,2, \ldots, N, \quad \nu=1,2, \ldots, V,
$$$$
\sum_{i=0}^{N} x_{i p \nu}-\sum_{j=0}^{N} x_{p j \nu}=0
$$$$
p=0,1,2, \ldots, N, \quad \nu=1,2, \ldots, V
$$$$
\sum_{i=1}^{N} q_{i} y_{i \nu} \leq Q_{\nu}, \quad \nu=1,2, \ldots, V
$$

$$
\begin{aligned}
& S T_{\nu} \geq y_{i \nu}\left(t_{i M}+p_{i M}\right), \\
& \quad i=1,2, \ldots, N, \quad \nu=1,2, \ldots, V, \\
& b_{i \nu} \geq y_{i \nu} * S T_{\nu}, \quad i=1,2, \ldots, N, \quad \nu=1,2, \ldots, V,
\end{aligned}
$$

$$
b_{0 \nu} \geq S T_{\nu} * \sum_{j=1}^{N} x_{0 j \nu}, \quad \nu=1,2, \ldots, V
$$

$$
\begin{aligned}
b_{i \nu} & +s_{i}+f_{i j \nu} \leq b_{j \nu} * x_{i j \nu}, \\
& i=0,1,2, \ldots, N, \quad j=1,2, \ldots, N, \quad \nu=1,2, \ldots, V
\end{aligned}
$$

$$
\begin{aligned}
& \operatorname{MRT} \geq x_{i 0 \nu}\left(b_{i \nu}+s_{i}+f_{i 0 \nu}\right), \\
& \quad i=1,2, \ldots, N, \quad \nu=1,2, \ldots, V
\end{aligned}
$$

$$
\begin{aligned}
& t_{i m}, b_{i \nu}, \text { MRT } \geq 0, \quad \forall i, m, \nu, \\
& Z_{i j}, y_{i \nu}, x_{i j \nu}, y y_{i j \nu}=\{0,1\}, \quad \forall i, j, \nu .
\end{aligned}
$$

The objective function minimizes MRT. It should be noted that $i=0$ or $j=0$ in the proposed model's formulation refers to shop center.

\subsection{Production scheduling constraints}

Constraint (1-1) ensures that only one job could be processed on a machine at any time; if $J_{i}$ precedes $J_{j}$, then $t_{i m}+p_{i m} \leq t_{j m}$; if $J_{j}$ proceeds $J_{i}, t_{j m}+$ $p_{j m} \leq t_{i m}$ for all operations of jobs on each machine. Constraint (1-2) ensures that $O_{j(m+1)}$ begins after completion of $O_{j m}$ on each machine.

\subsection{Distribution constraints}

Constraint (1-3) ensures that each job, $J_{i}$, is delivered only with one vehicle. Constraints (1-4) guarantee that each vehicle must depart from production center to only one customer in its route; indeed, each vehicle can be used once. Constraint (1-5) enforces that each customer is serviced by only one vehicle. Constraint (16 ) guarantees that each vehicle can cross paths between two customers ( $i$ and $j$ ) if that vehicle is allocated to both customers ( $i$ and $j$ ). Constraint (1-7) allows that, for each costumer, $p$, the entering vehicle must eventually leave this node. Constraint (1-8) ensures the sufficient capacity of vehicles.

\subsection{Integrated constraints}

Constraint (1-9) implies that vehicle starts routing after completion of all orders allocated to the vehicle in production center. Constraint (1-10) ensures that service time of each customer is after departure time of relevant vehicle. Constraint (1-11) implies the departure time of vehicles from production center. Constraint (1-12) implies that service time of each customer after the completion of previous customer service is the routing of allocated vehicle. In addition, Constraint (1-12) acts as a sub tour elimination constraint in this model. Constraint (1-13) is defined as the ending time of each route. The constraint in Eq. (1-13) ensures that the ending time at the delivery stage can be completed only after all vehicles return to the production center (or MRT that can be defined as maximum time of vehicles tour).

The obtained model is solved for small-sized instances using optimization software GAMS 23.8 and solver CPLEX 12.4. For medium- and large-sized instances, GAMS is a diagnosed inappropriate solution method for solving the proposed model due to nonacceptable solving time, thus meta-heuristics must be used for solving the proposed model. Finally, due to the advantages of ICA, ICA has been selected to solve the proposed integrated model. 


\section{Imperialist competitive algorithm}

\subsection{Imperialist competitive algorithm in general}

Imperialist Competitive Algorithm (ICA) is a kind of meta-heuristic algorithm used for solving different optimization problems. This algorithm, which was first proposed by Atashpaz and Lucas [35], is a population-based meta-heuristic inspired from sociopolitical behaviors. ICA begins with a random initial population. Each individual of the population is named a "country". In the first step of the algorithm, some of the best countries are selected as "imperialist countries", and the remaining countries are considered to be "colonies" of the imperialists.

Colonies are then divided among those imperialists based on their power. After dividing all colonies among the imperialist countries, the colonies move toward their relevant imperialists within the cultural state space in each iteration of algorithm. A collection of an imperialist country and some colonies is named an empire, competing among empires and displacing each other. The survival of an empire depends on its power to take over colonies from other competitors. The power of larger empires increases, while the empires of less power collapse in iterations. Following many iterations of competition among empires, the power of colonies approaches that of the imperialist countries, indicating convergence. The extreme bound of imperialist competition occurs when only one imperialist remains along with some colonies, which are close to the imperialist country in terms of position. Pseudo code of the ICA algorithm is shown in Figure 1.

There have been some reports on the applications of imperialist competitive algorithm in solving the scheduling problems by researchers [36-42]. Thus, based on proper reports of ICA for combinatorial and complicated problems, such as the developed model and appropriate performance of this algorithm, the authors have selected ICA for solving method. A review on the mentioned studies showed that this algorithm has not been used to solve permutation flowshop problems until now. In all these researches, ICA is stated as an effective algorithm. Therefore, another reason for selecting ICA by authors is the capability of ICA to solve permutation flow-shop problem.

\subsection{The Improved Imperialist Competitive Algorithm (I-ICA)}

Due to some improvement in the general ICA for solving the proposed model, the obtained algorithm was named as improved ICA (I-ICA) in this paper. One of the important issues of solving optimization problems using meta-heuristics is determining an appropriate

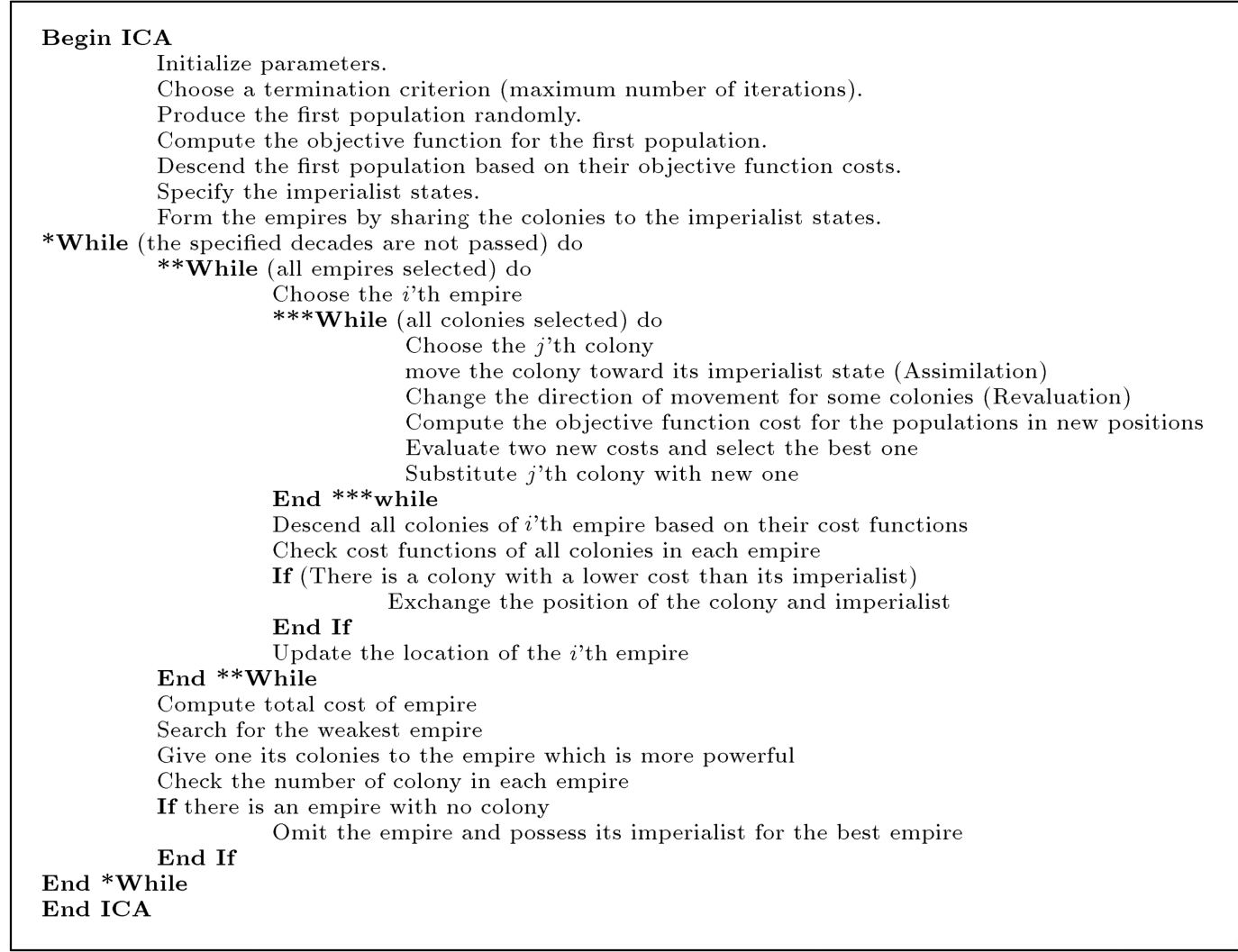

Figure 1. Pseudo code of the ICA algorithm. 


\begin{tabular}{l|llllll} 
Sol. Part 1 & 5 & 3 & 2 & 4 & 1 & \\
Sol. Part 2 | & 5 & 0 & 3 & 4 & 0 & 2
\end{tabular}

Figure 2. Two-part array for solution representation.

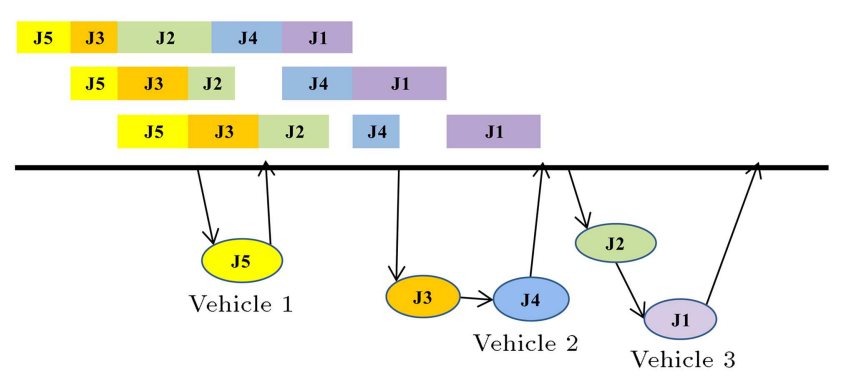

Figure 3. Example of country decoding.

format to represent a solution. In ICA, the term "country" is used for this array. Thus, we suggested an array with two parts of showing each solution (country). The first part refers to flow shop stage of solution, and the second part shows distribution with routing stage of solution as follows.

In the example shown in Figure 2, we have three machines in production stage, and there are five orders that must be produced in the sequence of 5-3-2-4-1 in all machines, and then delivered to their customers with three vehicles. Zeros in part two of country represent vehicle separator; for the sake of certainty, the feasible number of constraints of zero must be equal to $(V-1)$ and no two zeros placed behind each other. Figure 3 shows this example schematically.

The cost of a country is found by evaluating the cost function that defined MRT in this study:

$$
\text { Cost }=f(\text { country }) \text {. }
$$

\subsubsection{Generating initial empires}

In the initialization step, we need to generate an initial population with the size of $N_{\text {pop }}$. Next, we have to select some of the best countries with the lowest cost of function values and the size of $N_{\text {imp }}$ from $N_{\text {pop }}$ and set them to be imperialists. The rest of countries are set to be colonies $N_{\text {col }}\left(N_{\text {col }}=N_{\text {pop }}-N_{\text {imp }}\right)$.

Part one of country is random permutation of $1,2, \ldots, N$ numbers for flow shop scheduling solution generated completely randomly. For generating part two of country, three random policies are defined and proposed to obtain part two based on part one in a random approach, and then one random policy is defined to generate parts one and two in a random approach separately:

- Policy 1: Putting $(V-1)$ zeros between part 1 randomly to separate vehicles routines;

- Policy 2: Firstly, exchanging two positions of part 1 (swap), and then putting $(V-1)$ zeros between part 1 randomly to separate vehicles routines;

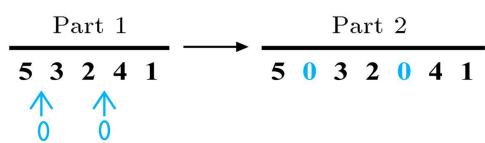

(a) Policy 1

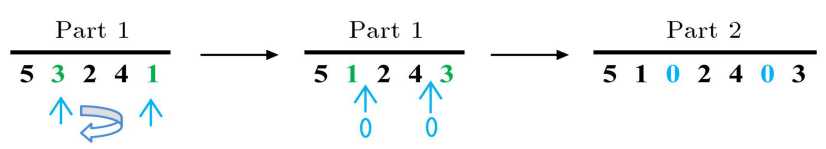

(b) Policy 2
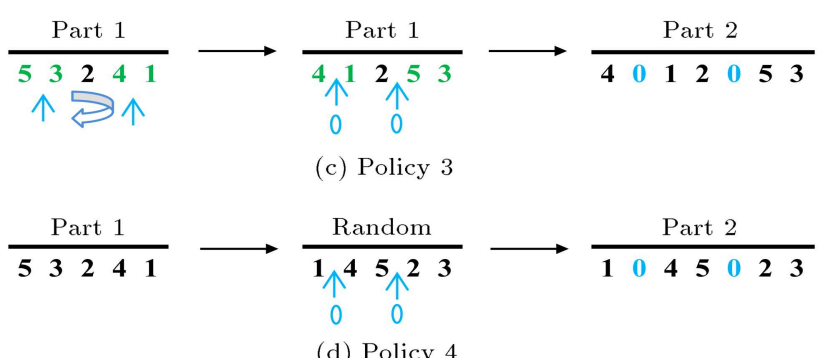

(d) Policy 4

Figure 4. Policies of initial population generation.

- Policy 3: Using multiple swaps for part 1, and then putting $(V-1)$ zeros between part 1 randomly to separate vehicles routines;

- Policy 4: Both parts one and two of solution scheme are generated based on random permutation of $1,2, \ldots, N$, and then $(V-1)$ zeros are inserted into part two randomly.

Figure 4 shows the policies for generating part 2 from part 1 of country.

Given the importance of initial solution quality in each algorithm, a certain percentage of the generated solutions used 2-opt neighbor search algorithm to enhance the quality of initial population. The 2-opt operator is used for intra-route improvement. In a 2-opt move, two non-adjacent arcs are replaced by another two new ones, and the visited order of the customers between the two arcs is reversed.

Considering initial population, the number of colonies of an empire is different based on the power of the imperialists. In other words, bigger empires have more colonies and smaller ones have less. The number of colonies for each empire is calculated by the following steps:

- Calculate the normalized cost of an imperialist $\left(t_{\max _{n}}\right)$ :

$$
C_{n}=\operatorname{Max}_{i}\left(t_{\max _{i}}\right)-t_{\max _{n}},
$$

where $t_{\max _{n}}$ is the makespan time of the $n$th imperialist;

- Calculate the normalized power of each imperialist $\left(P_{n}\right)$ :

$$
P_{n}=\left|\frac{C_{n}}{\sum_{i=1}^{N_{\text {imp }}} C_{i}}\right| \text {. }
$$


- Calculate the number of initial colonies for the $n$th empire $\left(N C_{n}\right)$ :

$$
N C_{n}=\operatorname{Round}\left\{P_{n} \cdot N_{\mathrm{Col}}\right\} \quad n=1,2, \ldots, n-1 .
$$

The following formula was developed for the last imperialist:

$$
N C_{n}=N_{\mathrm{col}}-\left(N C_{1}+N C_{2}+\ldots+N C_{n-1}\right) .
$$

\subsubsection{Assimilation: Moving the colonies of an empire toward the imperialist}

When empires are formed, imperialist states try to be more powerful by absorbing the other colonies. It means that colony moves toward the imperialist state in a random direction. In this step, also, three different policies are defined to assimilate colonies.

Policy 1: Putting two positions of parts 1 and 2 of an imperialist state identically in an assimilated array, bringing the rest from colony, and thus forming an assimilated colony (see Figure 5);

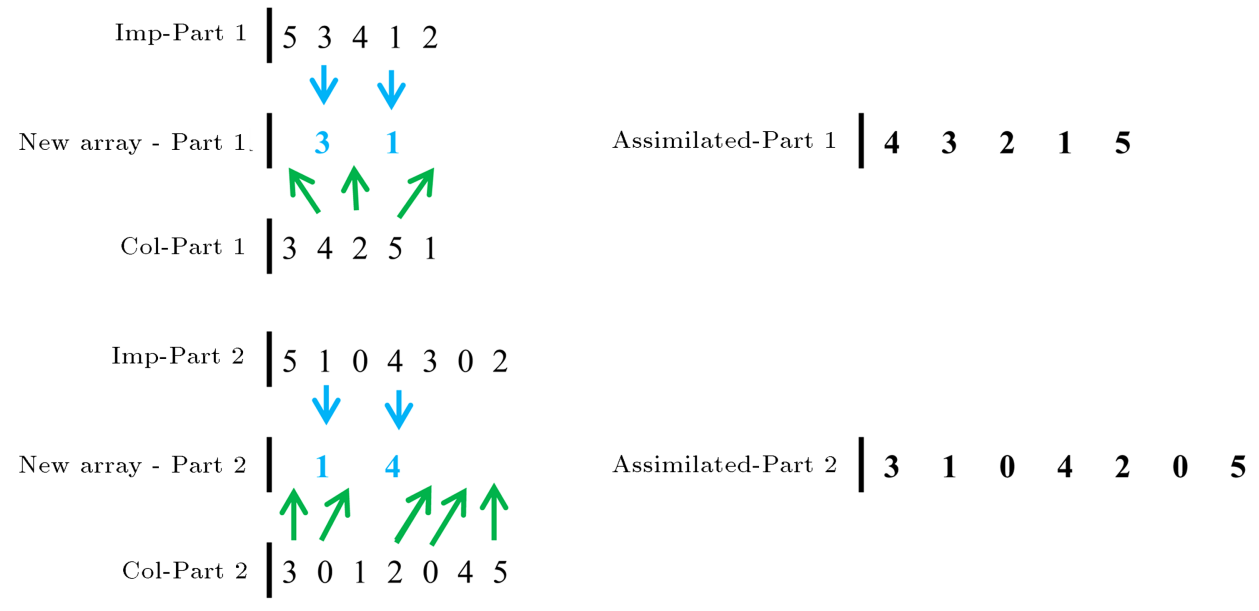

Figure 5. Policy 1 of assimilation.

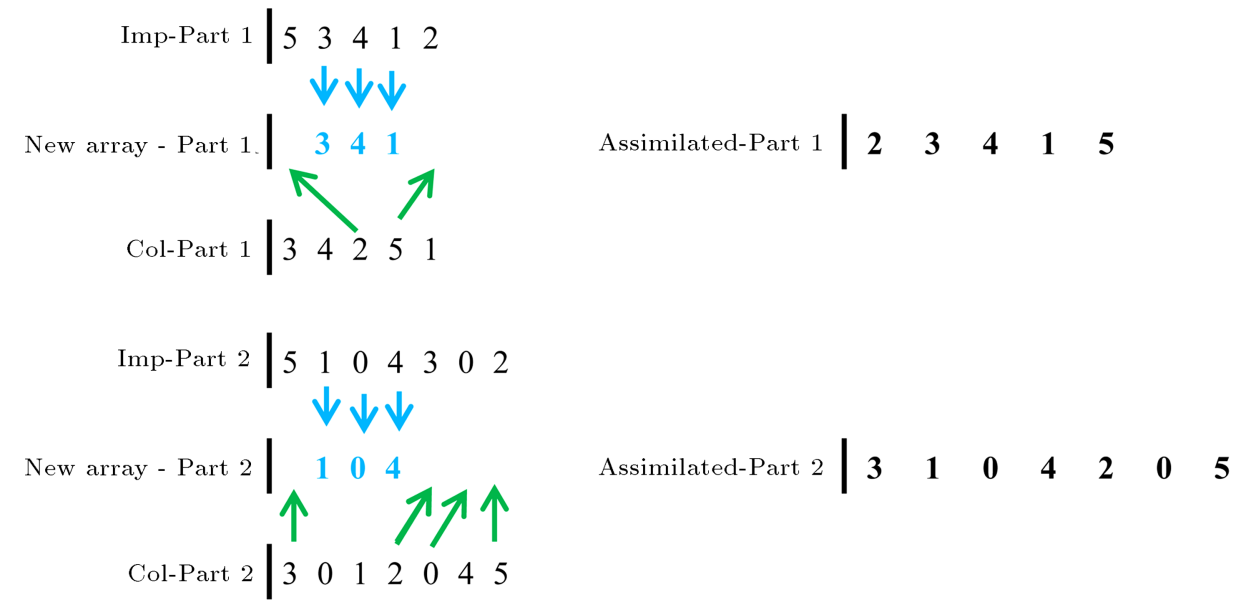

Figure 6. Policy 2 of assimilation.
Policy 2: Putting 1 subsequence of parts 1 and 2 of an imperialist state identically in an assimilated array, bringing the rest from colony, and thus forming an assimilated colony. The size of subsequence is linked to country size (see Figure 6);

Policy 3: Using Policy 1 for part 1 and Policy 2 for part 2 separately (see Figure 7 ).

\subsubsection{Revolution}

According to the imperial history, the imperialist absorbs colonies of an empire in terms of social, cultural, economic, and political characteristics; however, some colonies might resist being absorbed by imperialists. In fact, those colonies perform some sort of reformations in their characteristics. In ICA, this operation is called revolution. Revolution brings sudden random changes in the position of some colonies in the search space. Revolution operation in ICA increases exploration and prevents the early convergence of countries to local optima. By performing revolution operation, a colony suddenly jumps from one point to another random 


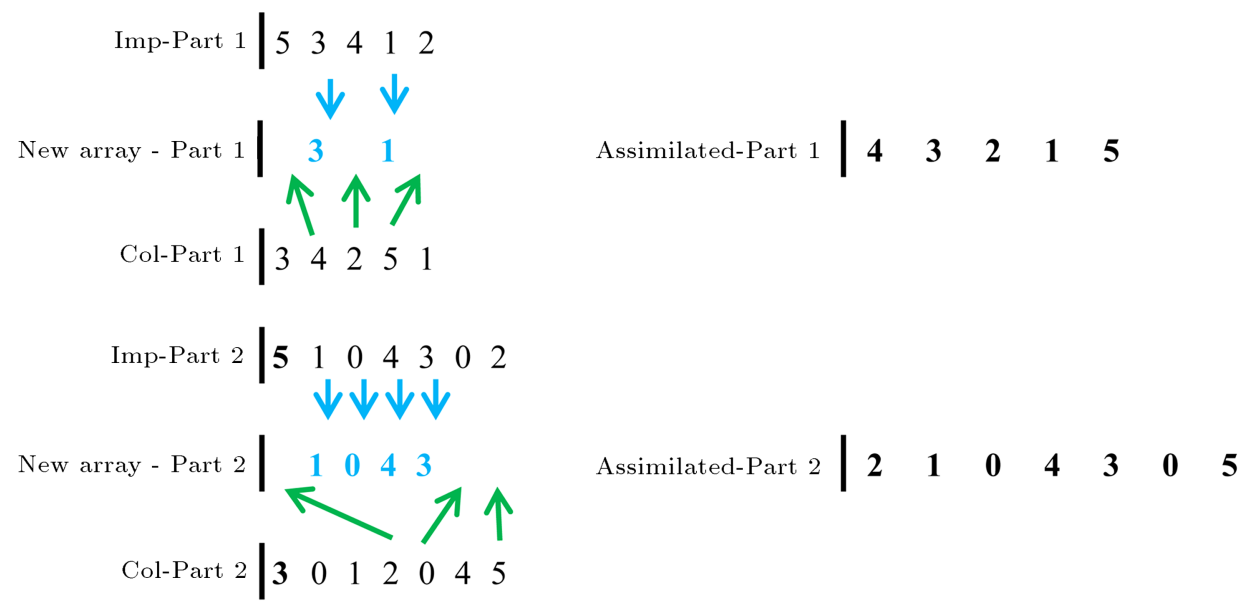

Figure 7. Policy 3 of assimilation.

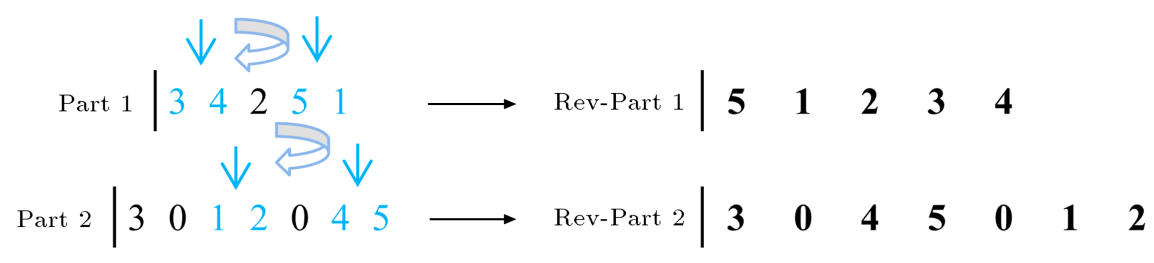

(a) Swap revolution method

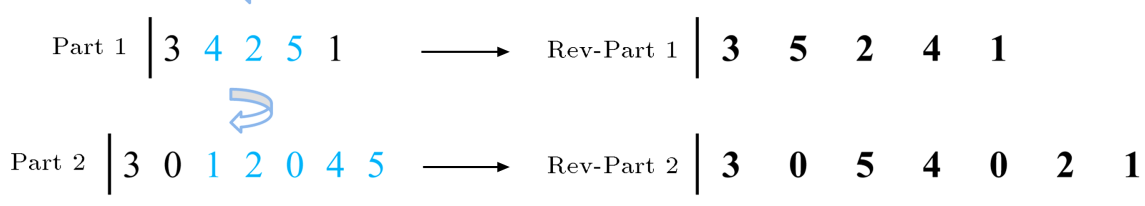

(b) Reversion revolution method

Figure 8. Revolution methods.

point which results in escaping from local optimum. In the proposed I-ICA for this step, three stage strategies are used. In the first strategy, only part one is changed by swap and reversion methods; in the second strategy, only part two is changed by 2-opt method, and finally, in the third strategy, parts one and two are changed simultaneously to revolutionize colonies. Choosing these three strategies is done randomly.

\section{Strategy 1:}

Swap procedure: In swap procedure, two arrays of part one containing two cells for each one are selected randomly, and the position of these arrays will be changed. Figure 8(a) shows the used swap procedure. Cells $\{3\}$ and $\{5\}$ are selected randomly in part 1 colony, and the new colony is obtained by replacing its positions.

Reversion procedure: For reversion procedure, a subsequence array from part one is selected randomly.
The position of the chosen cells is reversed, but the position of the array is unaffected. In Figure 8(b), the used reversion method is presented. Figure $8(\mathrm{~b})$ shows the used reversion procedure.

\section{Strategy 2:}

In this strategy, 2-opt operator is applied randomly to one vehicle route in part two. In a 2-opt move, two nonadjacent arcs are replaced by another two new ones, and the visited order of the customers between the two arcs is reversed. Figure 9 is an example of the above with arbitrary input;

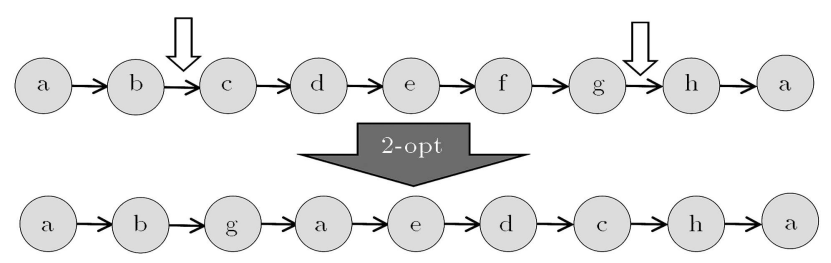

Figure 9. 2-opt strategy for revolution. 


\section{Strategy 3:}

In this strategy, Strategies 1 and 2 are used for parts one and two of solution scheme, respectively.

\subsubsection{Exchanging positions of the imperialist and a colony}

Once assimilation and revolution operations are performed on colonies of an empire, the cost functions of colonies' new positions are then compared with those of imperialists. If we find any colony whose cost function is less than that of imperialist, then we swap imperialist with that colony.

\subsubsection{Computing total power of an empire}

The power of an empire is computed based on the power of its imperialist and a fraction of the power of its colonies:

$$
\begin{aligned}
\mathrm{TC}_{n}= & \operatorname{Cost}\left(\operatorname{Imp}_{n}\right)+\varphi \\
& * \text { mean }\left\{\operatorname{cost}\left(\text { colonies of } \operatorname{Imp}_{n}\right)\right\},
\end{aligned}
$$

where $\mathrm{TC}_{n}$ is the total cost of the nth empire, and $\varphi$ is a positive number between 0 and 1 that is one of ICA parameters. A small value of $\varphi$ emphasizes a greater influence of imperialist power on determining the total power of empire, while a large value of $\varphi$ indicates the influence of the mean power of colonies in determining the total power of the empire.

\subsubsection{Imperialistic competition and eliminating the powerless empires}

During competition among imperialist countries, weaker empires will collapse gradually. Therefore, one or some of the weakest colonies belonging to the weakest empire will be given to a different empire based on competition that occurs among all empires. Stronger empires have a greater chance of possessing the weakest colony. In order to model the competition process among the empires, we need to compute the normalized total cost of empire as in the following:

$$
\mathrm{NTC}_{n}=\operatorname{Max}_{i}\left(\mathrm{TC}_{i}\right)-\mathrm{TC}_{n},
$$

where $\mathrm{TC}_{n}$ is the total cost of the $n$th empire, and $\mathrm{NTC}_{n}$ is the normalized total cost of the corresponding $n$th empire. Then, the probability of possessing a colony is computed as follows:

$$
P_{n}=\left|\frac{\mathrm{NTC}_{n}}{\sum_{i=1}^{N_{\mathrm{imp}}} \mathrm{NTC}_{i}}\right| .
$$

\section{Stopping criterion}

Many stopping criteria have been defined and developed in the literature so far. For example, it can be stopped after running a number of iterations called maximum decades or, when there is just one empire left. In this paper, it is decided to specify maximum decades as the stopping criterion.

\section{Repair procedure}

In different steps of ICA due to vehicles capacity constraint, some infeasible solutions may be obtained. In this section, repair procedure was proposed for these solutions. If one solution is infeasible in part 2 of a country, it only means that the capacity of one or more vehicles is more than that of a defined vehicle. Therefore, firstly, the zeros of part 2 are eliminated and customers are allocated to vehicles in order of remaining costumers. At the end, if the zeros in a new array are less than $(V-1)$, the remaining necessary zeros are placed randomly between non-zero members of an array (see Figure 10).

\section{Computational study}

This section describes the computational results obtained to investigate the performance of the proposed integrated model and I-ICA algorithm.

\subsection{Test problem}

Two types of scrutiny are involved in the computational study. First, the performance of the proposed algorithm is evaluated, and then the value of the developed integrated model is investigated. In order to evaluate the performance of algorithm, we compare our I-ICA algorithm with other solution methods. The authors use well-known PFSSP benchmark problems in literature and compare makespan criteria to evaluate the performance of the proposed algorithm.

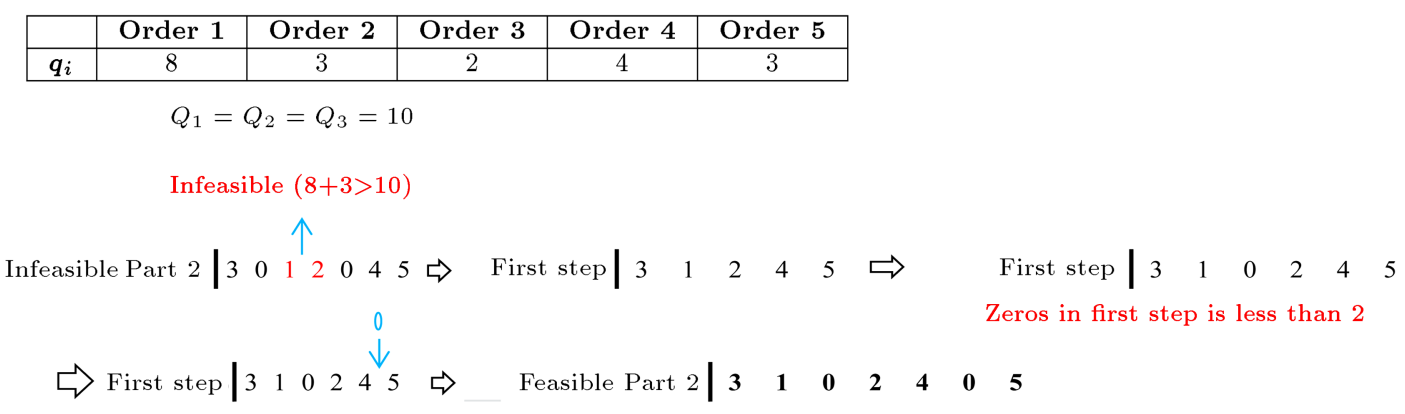

Figure 10. Example of repair procedure. 
On the other side, in order to investigate the value of integration by the developed model, some test problems are required. A careful set of instances is developed randomly since the authors are unaware of any available test problems for the proposed integrated model of flow-shop scheduling with routing decision to deliver orders to customers.

For this purpose, in flow-shop scheduling literature, three different types of well-known benchmark problems are considered. The sets of the first and second problems deal with the Carlier [43] and Reeves's [44] studies which can be obtained from the OR-Library, while sets of the third problem have been taken from Taillard's [45]. The above three instances are the famous flow-shop benchmark problems that are widely used in the production scheduling literature. Since the first integrated model of PFSSP and distribution with routing is proposed in this paper, the considered benchmarks do not completely match this problem. Hence, some modifications are needed. We use these three benchmarks as the data for production scheduling part of integrated model and generate the distribution with routing data randomly for each of production benchmarks in Table 1 .

In addition, the distance between customers defined with Euclidean distance and number of vehicles, $(V)$, could be defined as in the following function:

$$
V=\left[\frac{\text { Sum of customers' demands }}{\text { Vehicles capacity }(Q)}\right]+1 \text {. }
$$

\subsection{Parameter tuning}

The choice of parameters' value is a challenging task in each evolutionary algorithm, since it highly influences the quality of problem solutions. This subsection studies the effects of parameters on the proposed I-ICA algorithm. For tuning the parameters, there are several designs including full factorial experiment [46,47], Taguchi experiment [48], and so on. The Taguchi method is conducted in order to decrease the required tests. Taguchi method separates the factors into two main groups: controllable factors and noise factors. It assumes that there is no control on the noise factors, but we can optimize the controllable factors. Taguchi presents a variation measure called $\mathrm{S} / \mathrm{N}$ ratio (signal to noise). Herein, signal denotes the desirable value and noise denotes the undesirable value. The aim is to maximize the $\mathrm{S} / \mathrm{N}$ ratio to indicate how a parameter design is a robust design. Taguchi classified the objective function into three types: larger-the-better, smaller-the-better, and on-target, minimum-variation. The proposed integrated model is classified in the second type.

The control factors of this paper are the number of the countries and imperialist, the number of iterations, the revolution probability, and the influence of mean power of colonies on total power of empire. Different levels of these factors are shown in Table 2.

The associated degree of freedom is considered to be 9 ; therefore, the selected orthogonal array should have a minimum of 9 rows and four columns to

Table 1. Characteristics of distribution with routing test problems.

\begin{tabular}{ll}
\hline \multicolumn{1}{c}{ Factors } & Levels \\
\hline Depot location & $x_{\text {Depot }}=y_{\text {Depot }}=$ Uniform $(20,50)$ \\
Customers' location & $\left\{\begin{array}{l}x_{i \text { (Customer })}=\text { Uniform }\left(0,2 \times x_{\text {Depot }}\right) \\
y_{i \text { (Customer })}=\text { Uniform }\left(0,2 \times y_{\text {Depot }}\right)\end{array}\right.$ \\
Customers' demands $(d)$ & Uniform $(20,50)$ \\
Service time in customer location & Uniform $(0,5)$ \\
Number of vehicles $(V)$ & $f(d)$ function based on customers' demands \\
Vehicles capacity $(Q)$ & $\begin{cases}\text { Small }- \text { sized problem } & 40 \\
\text { Medium }- \text { sized problem } & 100 \\
\text { Large }- \text { sized problem } & 150\end{cases}$ \\
\hline
\end{tabular}

Table 2. Different levels of the factors.

\begin{tabular}{lcc}
\hline Factors & Symbols & Levels \\
\hline Number of the countries and imperialists & $\left(N_{\text {Col }}, N_{\text {Imp }}\right)$ & $(50,10),(100,30),(150,40)$ \\
Maximum number of iterations & Max Iter & $100,150,200$ \\
Revolution probability & Rev percent & $0.2,0.3,0.4$ \\
Influence of mean power of colonies on total power of empire & Zeta percent & $0.1,0.2,0.4$ \\
\hline
\end{tabular}




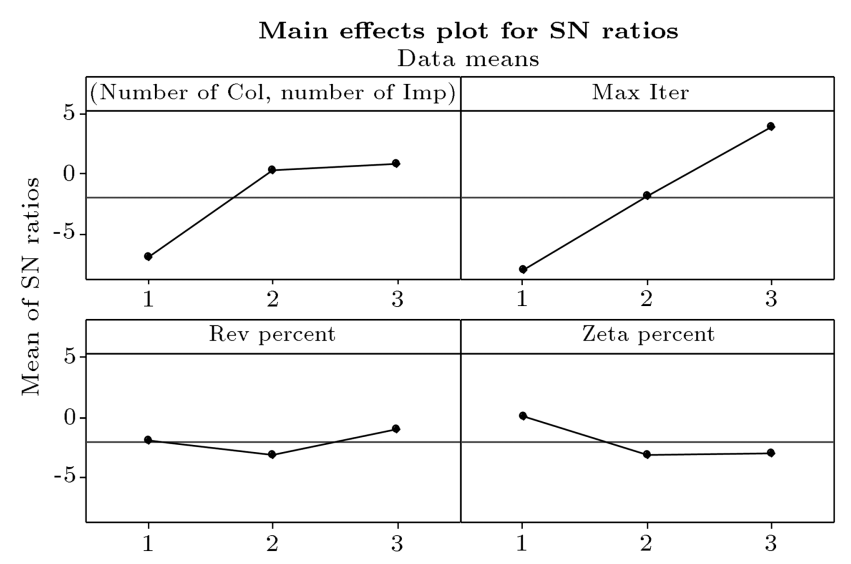

Signal-to-noise: Smaller is better

Figure 11. The S/N ratio plot for each level of factors.

accommodate the four factors. From the standard table of orthogonal arrays, L9 is selected as the fittest orthogonal array design that fulfills all our minimum requirements. Ten instances are solved for parameter setting as follows. We consider four smalland medium-sized instances from the first and second sets of problems which are randomly selected [43,44], and six medium- and large-sized instances from the third set of problems [45]. The Relative Percentage Deviation (RPD) is used as a performance measure to compare the methods. The RPD is calculated as follows:

$$
\mathrm{RPD}_{i, j}=\frac{x_{i, j}-x_{\min , j}}{x_{\min , j}} * 100,
$$

where $\mathrm{RPD}_{i, j}$ is the relative percentage deviation of the $j^{\prime}$ th instance of the $i^{\prime}$ th trial. $x_{i, j}$ is the objective function value that is obtained from the $j^{\prime}$ th of instance of the $i^{\prime}$ th trial. $x_{\min , j}$ is a minimum value of objective function obtained for $j^{\prime}$ th instance. When RPDs are calculated, the average of RPD of each trail is transformed to $\mathrm{S} / \mathrm{N}$ ratio. According to the $\mathrm{S} / \mathrm{N}$ ratio, the best level of each factor is determined. Figure 11 shows the main effects plot for $\mathrm{S} / \mathrm{N}$ ratio.

The selected levels of factors are as follows: $\left(N_{\mathrm{Col}}, N_{\mathrm{Imp}}\right)$ is equal to $(150,40)$, Max Iter is equal to 200 , Rev Percent is equal to 0.3 , and Zeta Percent is equal to 0.2 . In order to perform the experiments, the suggested algorithm was implemented in MATLAB 7.6.0.324 (R2008a) and run on a PC with $2.80 \mathrm{GHz}$ and 192 GB of RAM memory.

\subsection{Comparison method}

In this section, two important questions have been answered in computational study. First, does the proposed improved ICA algorithm have a suitable performance? Compared to hierarchical approach, does the integration approach improve the performance of the model? Alternatively, how much is the value of integration in the proposed model?
To answer the first question, the performance of the proposed I-ICA algorithm is compared with those of the four other algorithms in the literature (ODE, NEH, PGA, and HDDE) for solving PFSSP. ODE is a discrete differential evolution algorithm, PGA is a permutation-based genetic algorithm [49], NEH is a well-known constructive heuristic [50], and HDDE is a hybrid discrete differential evolution proposed by Mokhtari et al. [51]. For comparison, the four mentioned flow shop benchmarks are solved with improved ICA, and makespan is compared with other four algorithms solutions.

To answer the second question, the value of integration in the proposed model should be evaluated. For this purpose, distribution with routing data of integrated model is randomly generated based on each PFSSP benchmark, and then obtained benchmarks are solved by means of the proposed I-ICA algorithm; finally, hierarchical (approach) result of two models (flow shop and routing) is obtained. In order to evaluate the value of integration by the developed model, MRT is used. Based on MRT comparison, effectiveness of the proposed model is investigated where the obtained results show the good performance of integrated model. The hierarchical solution of the two models is equal to the sum of the solutions of two models separately solved by I-ICA. Then, the integrated model of flow shop and distribution with routing is solved by I-ICA, and solutions are compared with hierarchical ones. In this paper, statistical analysis using Rank-Based Friedman Test is proposed to evaluate value of integration in the proposed model.

\subsection{Experimental results}

The proposed I-ICA algorithm must be compared with other algorithms to evaluate the ICA ability in solving flow shop scheduling problem as well as developed integrated model.

Table 3 reports Carlier's benchmark results, while Reeves's, 20-job, and 50-job Taillard's benchmarks are shown in Tables 3-6, respectively.

The first two columns characterize the problem specifications in Table 3, while the third column represents the best-known solution reported for each problem in literature. The makespan measures, concerning ODE method PGA, NEH, and HDDE, are presented in the fourth, fifth, sixth, and seventh columns, respectively. The best value of makespan resulted from the improved ICA on Carlier problems is presented in the eighth column. The results shown in Table 3 revealed that our proposed I-ICA algorithm works better than the other four algorithms in optimizing Carliers problems. As we can see in Table 3, I-ICA can reach the best-known value of makespan in literature in all eight Carlier problems. As expected, I-ICA outperforms all other mentioned methods. Results 
Table 3. Computational results on Carliers' data sets.

\begin{tabular}{cccccccccc}
\hline Problem & $\boldsymbol{N} \times \boldsymbol{M}$ & $\begin{array}{c}\text { Best known } \\
\text { solution }\end{array}$ & ODE & PGA & NEH & HDDE & I-ICA & $\begin{array}{c}\text { Hierarchical } \\
\text { approach } \\
\text { MRT }\end{array}$ & $\begin{array}{c}\text { Integrated } \\
\text { approach } \\
\text { MRT }\end{array}$ \\
\hline Car1 & 11,3 & 7038 & 7038 & 7038 & 7038 & 7038 & $\mathbf{7 0 3 8}$ & 7115 & 7082 \\
Car2 & 13,4 & 7166 & 7166 & 7166 & 7376 & 7166 & $\mathbf{7 1 6 6}$ & 7250 & 7178 \\
Car3 & 12,5 & 7312 & 7366 & 7399 & 7399 & 7340 & $\mathbf{7 3 1 2}$ & 7410 & 7367 \\
Car4 & 14,4 & 8003 & 8003 & 8003 & 8003 & 8003 & $\mathbf{8 0 0 3}$ & 8090 & 8048 \\
Car5 & 10,6 & 7720 & 7720 & 7720 & 7835 & 7720 & $\mathbf{7 7 2 0}$ & 7799 & 7789 \\
Car6 & 8,9 & 8505 & 8505 & 8505 & 8773 & 8505 & $\mathbf{8 5 0 5}$ & 8620 & 8566 \\
Car7 & 7,7 & 6590 & 6590 & 6590 & 6590 & 6590 & $\mathbf{6 5 9 0}$ & 6684 & 6636 \\
Car8 & 8,8 & 8366 & 8366 & 8366 & 8564 & 8366 & $\mathbf{8 3 6 6}$ & 8467 & 8428 \\
\hline \multicolumn{2}{r}{ Average } & 7588 & 7594 & 7598 & 7697 & 7591 & $\mathbf{7 5 8 8}$ & 7679 & 7637 \\
\hline
\end{tabular}

Table 4. Computational results on Reeves' data sets.

\begin{tabular}{|c|c|c|c|c|c|c|c|c|c|}
\hline Problem & $N \times M$ & $\begin{array}{l}\text { Best known } \\
\text { solution }\end{array}$ & ODE & PGA & NEH & HDDE & I-ICA & $\begin{array}{c}\text { Hierarchical } \\
\text { approach } \\
\text { MRT } \\
\end{array}$ & $\begin{array}{c}\text { Integrated } \\
\text { approach } \\
\text { MRT }\end{array}$ \\
\hline Rec01 & 20,5 & 1247 & 1308 & 1283 & 1303 & 1260 & 1249 & 1371 & 1327 \\
\hline Rec03 & 20,5 & 1109 & 1117 & 1125 & 1132 & 1114 & 1109 & 1261 & 1203 \\
\hline Rec05 & 20,5 & 1242 & 1257 & 1245 & 1281 & 1245 & 1245 & 1377 & 1285 \\
\hline Rec07 & 20,10 & 1566 & 1599 & 1622 & 1626 & 1584 & 1568 & 1691 & 1660 \\
\hline Rec09 & 20,10 & 1537 & 1592 & 1587 & 1583 & 1582 & 1547 & 1686 & 1614 \\
\hline Rec11 & 20,10 & 1431 & 1494 & 1476 & 1550 & 1469 & 1445 & 1577 & 1524 \\
\hline Rec13 & 20,15 & 1930 & 2013 & 1975 & 2002 & 1975 & 1942 & 2062 & 2041 \\
\hline Rec15 & 20,15 & 1950 & 2028 & 2005 & 2013 & 2004 & 1971 & 2098 & 2076 \\
\hline $\operatorname{Rec} 17$ & 20,15 & 1902 & 2008 & 1960 & 2019 & 1956 & 1910 & 2017 & 2008 \\
\hline Rec19 & 30,10 & 2093 & 2230 & 2185 & 2185 & 2104 & 2104 & 2277 & 2229 \\
\hline Rec21 & 30,10 & 2017 & 2122 & 2113 & 2131 & 2091 & 2050 & 2177 & 2133 \\
\hline Rec23 & 30,10 & 2011 & 2130 & 2105 & 2155 & 2068 & 2028 & 2200 & 2188 \\
\hline $\operatorname{Rec} 25$ & 30,15 & 2513 & 2687 & 2654 & 2644 & 2631 & 2600 & 2777 & 2720 \\
\hline $\operatorname{Rec} 27$ & 30,15 & 2373 & 2503 & 2465 & 2498 & 2460 & 2413 & 2548 & 2532 \\
\hline Rec29 & 30,15 & 2287 & 2468 & 2459 & 2391 & 2389 & 2363 & 2551 & 2513 \\
\hline Rec31 & 50,10 & 3045 & 3291 & 3223 & 3173 & 3181 & 3148 & 3419 & 3365 \\
\hline Rec33 & 50,10 & 3114 & 3259 & 3206 & 3241 & 3177 & 3141 & 3385 & 3352 \\
\hline Rec35 & 50,10 & 3277 & 3356 & 3358 & 3313 & 3307 & 3280 & 3532 & 3459 \\
\hline $\operatorname{Rec} 37$ & 75,20 & 4951 & 5487 & 5299 & 5227 & 5255 & 5229 & 5515 & 5430 \\
\hline Rec39 & 75,20 & 5087 & 5542 & 5386 & 5308 & 5382 & 5362 & 5682 & 5553 \\
\hline Rec41 & 75,20 & 4960 & 5506 & 5332 & 5292 & 5344 & 5291 & 5543 & 5480 \\
\hline \multicolumn{2}{|c|}{ Average } & 2459 & 2619 & 2574 & 2575 & 2551 & 2524 & 2702 & 2652 \\
\hline
\end{tabular}

shown in Table 3 revealed the better performance of the integrated approach.

In order to give a better analysis, the RankBased Friedman Test is performed. For the statistical computations, we use the MINITAB 16 software environment. In this test, the null hypothesis $\left(H_{0}\right)$ is that the two sets of results (integrated and hierarchical approaches) come from the same population, and there is no significant difference between them. The chosen alpha level for this test is equal to 0.05. Thus, $H_{0}$ is rejected, and the effectiveness of the integrated approach changes:

$$
\begin{cases}H_{0}: & \text { Integrated approach }=\text { Hierarchical approach } \\ H_{1}: & \text { Otherwise }\end{cases}
$$

The results in Figure 12 indicate that integrated approach has a more efficient performance than hierarchical approach does.

The second dataset investigated in this section 


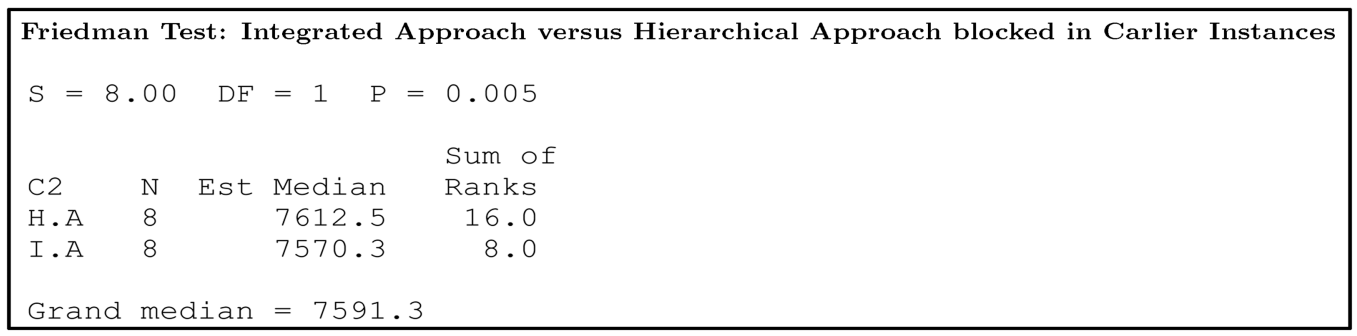

Figure 12. Comparison of integration and hierarchical approaches (Carlier set).

Table 5. Computational results on Taillard's benchmarks $\left(C_{\max }\right)(N=20)$.

\begin{tabular}{|c|c|c|c|c|c|c|c|c|}
\hline $\begin{array}{l}\text { Problem } \\
(N \times M)\end{array}$ & $\begin{array}{c}\text { Best known } \\
\text { solution }\end{array}$ & Palmer & CDS & NEH & HDDE & I-ICA & $\begin{array}{c}\text { Hierarchical } \\
\text { approach } \\
\text { MRT } \\
\end{array}$ & $\begin{array}{c}\text { Integrated } \\
\text { approach } \\
\text { MRT }\end{array}$ \\
\hline \multirow{10}{*}{$20 \times 5$} & 1278 & 1384 & 1390 & 1286 & 1278 & 1278 & 1413 & 1347 \\
\hline & 1359 & 1439 & 1424 & 1365 & 1360 & 1359 & 1484 & 1380 \\
\hline & 1081 & 1162 & 1249 & 1159 & 1123 & 1081 & 1234 & 1191 \\
\hline & 1293 & 1490 & 1418 & 1325 & 1317 & 1293 & 1419 & 1356 \\
\hline & 1235 & 1360 & 1323 & 1305 & 1265 & 1243 & 1370 & 1314 \\
\hline & 1195 & 1344 & 1312 & 1228 & 1224 & 1195 & 1326 & 1291 \\
\hline & 1239 & 1400 & 1393 & 1278 & 1251 & 1251 & 1411 & 1311 \\
\hline & 1206 & 1313 & 1341 & 1223 & 1220 & 1206 & 1327 & 1301 \\
\hline & 1230 & 1426 & 1360 & 1291 & 1271 & 1231 & 1336 & 1325 \\
\hline & 1108 & 1229 & 1164 & 1151 & 1132 & 1108 & 1248 & 1178 \\
\hline Average & 1222 & 1355 & 1337 & 1261 & 1244 & 1225 & 1357 & 1299 \\
\hline \multirow{10}{*}{$20 \times 10$} & 1582 & 1790 & 1757 & 1680 & 1637 & 1592 & 1711 & 1683 \\
\hline & 1659 & 1948 & 1854 & 1729 & 1711 & 1685 & 1834 & 1765 \\
\hline & 1496 & 1729 & 1645 & 1557 & 1556 & 1511 & 1622 & 1583 \\
\hline & 1377 & 1585 & 1547 & 1439 & 1419 & 1398 & 1535 & 1503 \\
\hline & 1419 & 1648 & 1558 & 1502 & 1496 & 1428 & 1543 & 1494 \\
\hline & 1397 & 1527 & 1591 & 1453 & 1446 & 1415 & 1534 & 1503 \\
\hline & 1484 & 1735 & 1630 & 1562 & 1526 & 1486 & 1620 & 1554 \\
\hline & 1538 & 1763 & 1788 & 1609 & 1599 & 1555 & 1691 & 1649 \\
\hline & 1593 & 1836 & 1720 & 1647 & 1644 & 1615 & 1749 & 1696 \\
\hline & 1591 & 1898 & 1884 & 1653 & 1637 & 1608 & 1738 & 1686 \\
\hline Average & 1514 & 1746 & 1697 & 1583 & 1567 & 1529 & 1658 & 1612 \\
\hline \multirow{10}{*}{$20 \times 20$} & 2297 & 2818 & 2559 & 2410 & 2376 & 2315 & 2453 & 2440 \\
\hline & 2099 & 2331 & 2285 & 2150 & 2142 & 2124 & 2245 & 2189 \\
\hline & 2326 & 2678 & 2565 & 2411 & 2387 & 2347 & 2442 & 2414 \\
\hline & 2223 & 2629 & 2434 & 2262 & 2261 & 2250 & 2398 & 2346 \\
\hline & 2291 & 2704 & 2506 & 2397 & 2383 & 2327 & 2456 & 2414 \\
\hline & 2226 & 2572 & 2422 & 2349 & 2280 & 2243 & 2389 & 2343 \\
\hline & 2273 & 2456 & 2489 & 2362 & 2345 & 2290 & 2429 & 2413 \\
\hline & 2200 & 2435 & 2362 & 2249 & 2249 & 2216 & 2362 & 2313 \\
\hline & 2237 & 2754 & 2414 & 2320 & 2319 & 2253 & 2383 & 2357 \\
\hline & 2178 & 2633 & 2469 & 2277 & 2270 & 2197 & 2297 & 2265 \\
\hline Average & 2235 & 2601 & 2451 & 2319 & 2301 & 2256 & 2385 & 2349 \\
\hline
\end{tabular}


Table 6. Computational results on Taillard's benchmarks $\left(C_{\max }\right)(N=50)$.

\begin{tabular}{|c|c|c|c|c|c|c|c|c|}
\hline $\begin{array}{l}\text { Problem } \\
(N \times M)\end{array}$ & $\begin{array}{l}\text { Best known } \\
\text { solution }\end{array}$ & Palmer & CDS & NEH & HDDE & I-ICA & $\begin{array}{c}\text { Hierarchical } \\
\text { approach } \\
\text { MRT } \\
\end{array}$ & $\begin{array}{c}\text { Integrated } \\
\text { approach } \\
\text { MRT }\end{array}$ \\
\hline \multirow{10}{*}{$50 \times 5$} & 2724 & 2774 & 2816 & 2733 & 2729 & 2729 & 2970 & 2904 \\
\hline & 2834 & 3041 & 3032 & 2843 & 2862 & 2848 & 3057 & 2994 \\
\hline & 2621 & 2777 & 2703 & 2640 & 2644 & 2621 & 2902 & 2811 \\
\hline & 2751 & 2860 & 2884 & 2782 & 2789 & 2770 & 2986 & 2908 \\
\hline & 2863 & 2963 & 3038 & 2868 & 2864 & 2864 & 3114 & 3007 \\
\hline & 2829 & 3090 & 3031 & 2850 & 2835 & 2832 & 3030 & 2936 \\
\hline & 2725 & 2845 & 2969 & 2758 & 2746 & 2741 & 2982 & 2932 \\
\hline & 2683 & 2826 & 2835 & 2721 & 2715 & 2710 & 2958 & 2855 \\
\hline & 2552 & 2733 & 2784 & 2576 & 2578 & 2569 & 2813 & 2716 \\
\hline & 2782 & 2915 & 2942 & 2790 & 2784 & 2783 & 3008 & 2913 \\
\hline Average & 2736 & 2882 & 2903 & 2756 & 2755 & 2747 & 2982 & 2898 \\
\hline \multirow{10}{*}{$50 \times 10$} & 2991 & 3478 & 3421 & 3135 & 3126 & 3082 & 3333 & 3273 \\
\hline & 2867 & 3313 & 3246 & 3032 & 2991 & 2986 & 3216 & 3114 \\
\hline & 2839 & 3321 & 3280 & 2986 & 2989 & 2968 & 3215 & 3142 \\
\hline & 3063 & 3511 & 3393 & 3198 & 3178 & 3142 & 3343 & 3308 \\
\hline & 2976 & 3427 & 3375 & 3160 & 3138 & 3091 & 3351 & 3334 \\
\hline & 3006 & 3323 & 3400 & 3178 & 3151 & 3114 & 3344 & 3266 \\
\hline & 3093 & 3457 & 3520 & 3277 & 3237 & 3176 & 3415 & 3373 \\
\hline & 3037 & 3356 & 3387 & 3123 & 3152 & 3120 & 3376 & 3316 \\
\hline & 2897 & 3414 & 3251 & 3002 & 2991 & 2986 & 3226 & 3170 \\
\hline & 3065 & 3404 & 3429 & 3257 & 3222 & 3152 & 3417 & 3411 \\
\hline Average & 2983 & 3400 & 3370 & 3135 & 3118 & 3082 & 3324 & 3271 \\
\hline \multirow{10}{*}{$50 \times 20$} & 3771 & 4272 & 4328 & 4082 & 4067 & 4025 & 4274 & 4218 \\
\hline & 3668 & 4303 & 4216 & 3921 & 3900 & 3893 & 4095 & 4062 \\
\hline & 3591 & 4210 & 4189 & 3927 & 3989 & 3852 & 4118 & 4081 \\
\hline & 3635 & 4233 & 4280 & 3969 & 3962 & 3931 & 4173 & 4094 \\
\hline & 3553 & 4376 & 4122 & 3835 & 3868 & 3817 & 4062 & 3978 \\
\hline & 3667 & 4312 & 4267 & 3914 & 3913 & 3905 & 4125 & 4073 \\
\hline & 3672 & 4306 & 4134 & 3952 & 3938 & 3907 & 4171 & 4138 \\
\hline & 3627 & 4310 & 4262 & 3938 & 3960 & 3924 & 4168 & 4137 \\
\hline & 3645 & 4547 & 4212 & 3952 & 4014 & 3939 & 4244 & 4145 \\
\hline & 3696 & 4197 & 4270 & 4079 & 4146 & 3907 & 4155 & 4042 \\
\hline Average & 3653 & 4307 & 4228 & 3957 & 3976 & 3910 & 4159 & 4097 \\
\hline
\end{tabular}

concerns Reeves' benchmarks in Table 4. The first column in Table 4 indicates the problem names. The second column specifies the size of each problem. The fourth to seventh columns represent the best makespan resulted from ODE, PGA, NEH, and HDDE, respectively. The 8 th columns represent the best makespan obtained from the I-ICA algorithm. After comparing the I-ICA with the mentioned algorithms having seemingly better results, the distribution with routing problem is also solved by I-ICA separately. The sum of these two solutions is named as MRT for hierarchical approach of PFSSP and distribution with routing, and the results are shown in the 9th column. At the end, the proposed integrated model was 


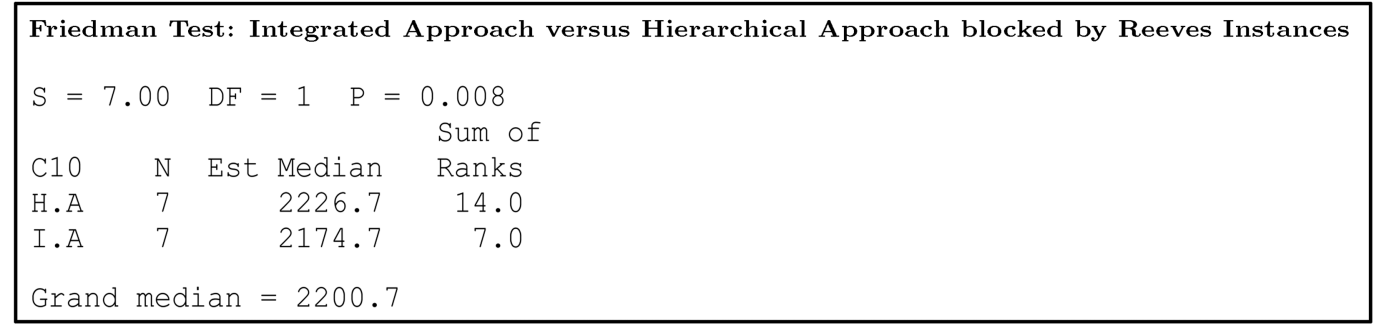

Figure 13. Comparison of integrated and hierarchical approaches (Reeves' set).

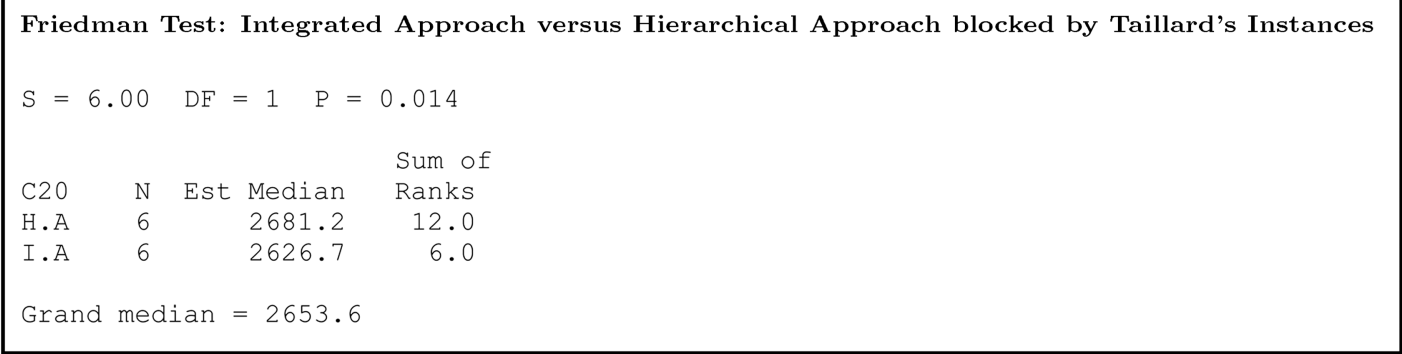

Figure 14. Comparison of integrated and hierarchical approaches (Reeves'set).

solved by the proposed modified ICA algorithm, and MRT results are reported in 10th column of Table 4. By comparing the 9th and 10th columns' results, we can see the better performance of integrated approach clearly rather than hierarchical approach.

As we can see in Table 4, I-ICA can reach the bestknown value of makespan in one Reeves' problem. As expected, I-ICA outperforms all other four mentioned methods. According to the attained results, the solutions obtained by I-ICA are better than those obtained by ODE, PGA, NEH, and HDDE are for all instances, respectively.

Meanwhile, the average MRT obtained from IICA for the integrated approach is only 2652, whereas the average value of MRT calculated by the hierarchical approach is 2702, respectively. The results shown in Table 4 revealed the better performance of the integrated approach. By using the mentioned RankBased Friedman Test, we defined each of the three problems in Revees' instance with the same size as one block, and average of these three problems is used for Freidman test. The results in Figure 13 indicate that the integrated approach, unlike the hierarchical approach, has a more efficient performance for the problem.

The third and fourth datasets investigated in this section concern Taillard's benchmarks. In Tables 5 and 6 , the best attained results of the presented I-ICA on both original and modified Taillard's benchmarks are presented, and they are compared with the three well-known heuristics, namely Palmer's index (Palmer 1965), CDS (Campbell et al., 1970 [52]), and NEH (Nawaz et al., 1983 [50]) and one metaheuristic namely HDDE (Mokhtari et al., 2010 [51]). The third column represents an upper bound for each problem obtained from Taillard's website. The last two columns indicate the performances of the integrated and hierarchical approaches.

As the results presented in Tables 5 and 6 show, the I-ICA outperforms other algorithms in all sets of problems and obtains the best-known solution to seven problems (30 out of all problems). The solutions of IICA are better than those by the Palmer, CDS, NEH, and HDDE methods are for all instances. Furthermore, the average deviation from the upper bound obtained by the suggested methods is less than the other four algorithms. With regard to the other set of Taillard's problems shown in Tables 5 and 6 , the average makespan obtained by I-ICA is less than those by other four methods are. As can be seen, the results obtained by I-ICA are equal to or better than those by the Palmer, CDS, and HDDE methods are for all the instances. By using the mentioned Rank-Based Friedman Test, we defined each of ten problems in Taillard's instance with the same size as one block, and average of these ten problems is used for Friedman test. The results in Figure 14 indicate that the integrated approach has a more efficient performance for the problem.

\section{Managerial insight}

Various types of wood products orders by customers who are in different locations will be sent to the production plant. These orders include different models of adult and children bed sets, a wide variety of home wood decorative, different models of TV tables, different models of shelves for shoes, etc. Several reputable companies with experience in this industry are 
working in Iran. Different workstations are located in manufacturing plants to arrange activities on products from raw materials to final products. Sheet cutting station, PVC covers station, etc. are embedded usually in these factories. Different orders are processed at all stations in order to be ready to be sent to the relevant customers. Thus, a flow shop system can be used to describe the mentioned production environment. After completion of the production, orders must be loaded and departed from plant to the different customers. Routing approach is used for delivery of orders by capacitated vehicles.

Production and delivery schedules are usually done separately in these factories. At certain times of the year when orders are faced with a significant increase in demand for products, the customers will encounter long delays in delivery. Based on management review, this delay reduces customer satisfaction, reduces readmissions dis-satisfied customers, and ultimately reduces profitability. Integrated model presented in this study has been proposed as a way to manage this issue for manufacturing environments. According to the mentioned production system and routing delivery method for transporting the orders by vehicles, employment of the integrated model presented in this paper could be proposed to managers as an effective and efficient solution (completion time is defined as the time when an order is delivered to the destination).

To clarify the real application of the proposed model in wood industry, consider 22 customers' zone from 22 different districts of Tehran urban area with different orders (see Figure 15). The production plant is located in one of industrial complex around of Tehran. Five stations are working in the manufacturing sector: Sheet Cutting station (SC), PVC covers station (PVC), Initial Assembly station (IA), Final Assembly station (FA), and Packaging station (P).

Customers and products data are shown in Ta-

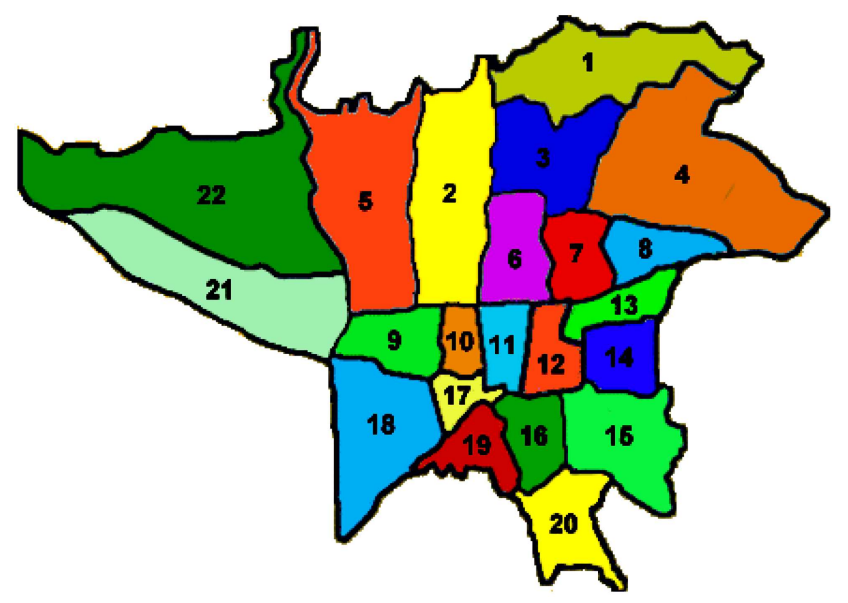

Figure 15. The customers' zones in Tehran. ble 7 . The location of production center ( $X, Y$ axes) is $(350,350)$, and 5 vehicles with capacity of 100 are used to deliver orders.

The proposed model is implemented based on the above data. The optimal solution is shown in Table 8. The total cost is equal to 2320 using the proposed model, optimal production schedule is shown in the second row, and the best vehicles routing for this problem are shown in the last five rows of Table 8 .

For evaluation of system performance, five plans have been considered as follows:

1. Plan 1: Replace existing vehicles with 3 new vehicles having a capacity of 200 ;

2. Plan 2: Replace existing vehicles with 7 vehicles having a capacity of 85 ;

3. Plan 3: Repair existing vehicles and increase their speed by $10 \%$;

4. Plan 4: $30 \%$ reduction in processing time of stations $1,2,5$;

5. Plan 5: 20\% reduction in assembly time (stations $3,4)$.

In order to analyze the sensitivity of the model, comprehensive analysis is presented for the following five managers' improvement plan. After making modifications to the model in any plan, a new problem was solved by ICA whose results are shown in Table 9 .

Based on the results shown in Table 9, implementation of the first plan will increase objective value by as much as 3.8 percent, because it can increase the length of routes traveled by vehicles. Thus, this plan could be selected only if a significant reduction occurred in the total cost of system by decreasing vehicles. In the following, the second, third, fourth, and fifth planes improve the objective values by 10.2 , $14.2,6.9$, and 3.7 percent, respectively. Based on the results, the amount of improvement of the objective function by implementing plan three (overhauling the current vehicles) has led to the greatest reduction in the objective values.

\section{Conclusions and further research}

In this investigation, the aim was to develop a new integrated model for a flow shop and distributionscheduling problem. In the proposed model, a permutation flow shop scheduling was assumed for production system, and a distribution with routing was assumed for delivery system. The objective was to minimize the Maximum Returning Time (MRT) for integrated model, meaning that the time of the last order delivered to relevant customer by the last vehicle and that vehicle returning to production center. In order to solve the problem, the imperialist competitive algorithm (IICA) was proposed. To enhance the performance of 
Table 7. Customers' location and orders data.

\begin{tabular}{ccccccccc}
\hline Customer/order & $\begin{array}{c}\text { SC } \\
\text { station }\end{array}$ & $\begin{array}{c}\text { PVC } \\
\text { station }\end{array}$ & $\begin{array}{c}\text { IA } \\
\text { station }\end{array}$ & $\begin{array}{c}\text { FA } \\
\text { station }\end{array}$ & $\begin{array}{c}\boldsymbol{P} \\
\text { station }\end{array}$ & $\begin{array}{c}\text { Location } \\
(\boldsymbol{X} \text { axis })\end{array}$ & $\begin{array}{c}\text { Location } \\
\text { (Y axis })\end{array}$ & $\begin{array}{c}\text { Occupied } \\
\text { space }\end{array}$ \\
\hline $\mathbf{1}$ & 27 & 79 & 22 & 93 & 38 & 220 & 550 & 15 \\
$\mathbf{2}$ & 92 & 23 & 93 & 22 & 84 & 380 & 340 & 29 \\
$\mathbf{3}$ & 75 & 66 & 62 & 64 & 62 & 390 & 380 & 37 \\
$\mathbf{4}$ & 94 & 5 & 53 & 81 & 10 & 450 & 610 & 36 \\
$\mathbf{5}$ & 18 & 15 & 30 & 94 & 11 & 420 & 690 & 3 \\
$\mathbf{6}$ & 41 & 51 & 34 & 97 & 93 & 110 & 10 & 1 \\
$\mathbf{7}$ & 37 & 2 & 27 & 54 & 57 & 290 & 250 & 23 \\
$\mathbf{8}$ & 58 & 81 & 30 & 82 & 81 & 260 & 370 & 2 \\
$\mathbf{9}$ & 56 & 12 & 54 & 11 & 10 & 60 & 520 & 8 \\
$\mathbf{1 0}$ & 20 & 40 & 77 & 91 & 40 & 170 & 680 & 1 \\
$\mathbf{1 1}$ & 2 & 59 & 24 & 23 & 62 & 700 & 490 & 32 \\
$\mathbf{1 2}$ & 39 & 32 & 47 & 32 & 49 & 640 & 190 & 8 \\
$\mathbf{1 3}$ & 91 & 16 & 39 & 26 & 90 & 550 & 670 & 38 \\
$\mathbf{1 4}$ & 81 & 87 & 66 & 22 & 34 & 90 & 640 & 31 \\
$\mathbf{1 5}$ & 33 & 78 & 41 & 12 & 11 & 390 & 320 & 33 \\
$\mathbf{1 6}$ & 14 & 41 & 46 & 23 & 81 & 40 & 510 & 17 \\
$\mathbf{1 7}$ & 88 & 43 & 24 & 34 & 51 & 470 & 390 & 12 \\
$\mathbf{1 8}$ & 22 & 94 & 23 & 87 & 21 & 580 & 700 & 27 \\
$\mathbf{1 9}$ & 36 & 1 & 68 & 59 & 39 & 640 & 270 & 34 \\
$\mathbf{2 0}$ & 65 & 93 & 50 & 2 & 27 & 100 & 620 & 35 \\
$\mathbf{2 1}$ & 25 & 35 & 17 & 12 & 23 & 650 & 510 & 5 \\
\end{tabular}

Table 8. Customers' location and orders data.

\begin{tabular}{ll}
\hline Objective value & 2320 \\
Production scheduling & $21-6-22-12-7-16-20-4-11-2-1-18-19-8-3-14-13-15-5-17-9-10$ \\
& Vehicle $1: 20-10-2-15$ \\
& Vehicle 2: $12-19-11-1$ \\
Delivery scheduling & Vehicle $3: 5-13-18-21$ \\
& Vehicle 4: $4-16-6-7$ \\
& Vehicle 5: $3-17-22-14-9-8$ \\
\hline
\end{tabular}

Table 9. Sensitivity analysis of the studied problem.

\begin{tabular}{|c|c|c|}
\hline Plan & Objective value & \% Improvement \\
\hline Plan 1 (decrease vehicles) & 2409 & -3.8 \\
\hline Plan 2 (increase vehicles) & 2080 & 10.3 \\
\hline Plan 3 (repair vehicles) & 1991 & 14.2 \\
\hline Plan 4 (replace production machines) & 2160 & 6.9 \\
\hline Plan 5 (increase production workforce) & 2242 & 3.7 \\
\hline
\end{tabular}

the suggested algorithm, some effective policies are proposed for improving the ICA performance:

- Three policies for generating initial empires;

- Three policies for assimilation;

- Two procedures for revolution;

- New repair procedure for infeasible solutions.
The original ICA was renamed to I-ICA algorithm based on these four main changes in original ICA. Three proper and useful sets of test problems were generated randomly and used for computational study. Taguchi method was used for the proposed algorithm parameter tuning. Computational results done on benchmark problems showed the effectiveness of integration of the proposed model and suggested 
approach with regard to the other methods. The useful statistical tool is used to evaluate the value of integration performance of the proposed model. One of the more significant findings to emerge from this study is that the integration of scheduling with delivery leads to the improvement of model performance. In this study, all parameters of the proposed model are deterministic; developing stochastic model could be a study case for further research. As another direction for future research, it could be considered to apply other new efficient meta-heuristic algorithms, such as bat algorithm, cuckoo search algorithm, neural network, and firefly algorithm, to this problem to obtain better solutions. In addition, other performance measures, such as mean tardiness, maximum tardiness, and mean completion time, can be considered instead of the MRT criterion. Other distribution approaches could be suggested in integrated model instead of distribution with routing. It would be valuable to extend this paper to allow for some other cost definitions or some other manufacturing environments including flow shops and job shops.

\section{References}

1. Chang, Y.C. and Lee, C.Y. "Machine scheduling with job delivery coordination", European Journal of Operational Research, 158, pp. 470-487 (2004).

2. Potts, C.N. "Analysis of a heuristic for one machine sequencing with release dates and delivery times", Operations Research, 28, pp. 1436-1441 (1980).

3. Hall, L.A. and Shmoys, B. "Jackson's rule for singlemachine scheduling: Making a good heuristic better", Mathematics of Operations Research, 17, pp. 22-35 (1992).

4. Matsuo, H. "The weighted total tardiness problem with fixed shipping times and overtime utilization", Operations Research, 36, pp. 293-307 (1988).

5. Herrmann, J.W. and Lee, C.Y. "On scheduling to minimize earliness-tardiness and batch delivery costs with a common due date", European Journal of Operational Research, 70, pp. 272-288 (1993).

6. Yuan, J. "A note on the complexity of singlemachine scheduling with a common due date, earlinesstardiness, and batch delivery costs", European Journal of Operational Research, 94, pp. 203-205 (1996).

7. Chen, Z.L. "Scheduling and common due date assignment with earliness-tardiness penalties and batch delivery costs", European Journal of Operational Research, 93, pp. 49-60 (1996).

8. Cheng, T.C.E., Gordon, V.S., and Kovalyov, M.Y. "Single machine scheduling with batch deliveries", European Journal of Operational Research, 94, pp. 277-283 (1996).

9. Yang, X. "Scheduling with generalized batch delivery dates and earliness penalties", IIE Transactions, 32, pp. $735-741$ (2000).
10. Hall, N.G., Lesaoana, M.A., and Potts, C.N. "Scheduling with fixed delivery dates", Operations Research, 49, pp. 134-144 (2001).

11. Hall, N.G. and Potts, C.N. "Supply chain scheduling: Batching and delivery", Working Paper, Department of Management Sciences, The Ohio State University, Columbus, OH (2000).

12. Lee, C.Y. and Chen, Z.L. "Machine scheduling with transportation considerations", Journal of Scheduling, 4, pp. 3-24 (2001).

13. Hall, N.G. and Potts, C.N. "Supply chain scheduling: batching and delivery", Operations Research, 51, pp. 566-584 (2003).

14. Garcia, J.M., Lozano, S., and Canca, D. "Coordinated scheduling of production and delivery from multiple plants", Robotics and Computer-Integrated Manufacturing, 20, pp. 191-198 (2004).

15. Li, C.L., Vairaktarakis, G., and Lee, C.Y. "Machine scheduling with deliveries to multiple customer locations", European Journal of Operational Research, 164, pp. 39-51 (2005).

16. He, Y., Zhong, W., and Gu, H. "Improved algorithms for two single machine scheduling problems", Theoretical Computer Science, 363, pp. 257-265 (2006).

17. Zhong, W.Y., Dosa, G., and Tan, Z.Y. "On the machine scheduling problem with job delivery coordination", European Journal of Operational Research, 182, pp. 1057-1072 (2007).

18. Su, C.Sh., Pan, J.C.H., and Hsu, T.S. "A new heuristic algorithm for the machine scheduling problem with job delivery coordination", Theoretical Computer Science, 410, pp. 2581-2591 (2009).

19. Li, C.L. and Vairaktarakis, G.L. "Coordinating production and distribution of jobs with bundling operations", IIE Transactions, 39, pp. 203-215 (2007).

20. Wang, X.L. and Cheng, T.C.E. "Machine scheduling with an availability constraint and job delivery coordination", Naval Research Logistics, 54, pp. 11-20 (2007).

21. Chen, B. and Lee, C.Y. "Logistics scheduling with batching and transportation", European Journal of Operational Research, 189, pp. 871-876 (2008).

22. Lu, L., Yuang, J., and Zhang, L. "Single machine scheduling with release dates and job delivery to minimize the makespan", Theoretical Computer Science, 393, pp. 102-108 (2008).

23. Liu, P. and Lu, X. "An improved approximation algorithm for single machine scheduling with job delivery", Theoretical Computer Science, 412, pp. 270-274 (2011).

24. Woeginger, G.J. "Heuristics for parallel machine scheduling with delivery times", Acta Informatica, 31, pp. 503-512 (1994). 
25. Wang, G. and Cheng, T.C.E. "Parallel machine scheduling with batch delivery costs", International Journal of Production Economics, 68, pp. 177-183 (2000).

26. Hall, N.G. and Potts, C.N. "The coordination of scheduling and batch deliveries", Annals of Operations Research, 135, pp. 41-64 (2005).

27. Chen, Z.L. and Vairaktarakis, G.L. "Integrated scheduling of production and distribution operations", Management Science, 51, pp. 614-628 (2005).

28. Ullrich, C.A. "Integrated machine scheduling and vehicle routing with time windows", European Journal of Operational Research, 227, pp. 152-165 (2013).

29. Wan, L. and Zhang, A. "Coordinated scheduling on parallel machines with batch delivery", International Journal of Production Economics, 150, pp. 199-203 (2014).

30. Lee, I.S. "A coordinated scheduling of production-anddelivery under dynamic delivery cost environments", Computers \& Industrial Engineering, 81, pp. 22-25 (2015).

31. Lu, L., Zhang, L., and Wan, L. "Integrated production and delivery scheduling on a serial batch machine to minimize the makespan", Theoretical Computer Science, 572, pp. 50-57 (2015).

32. Yuan, J., Soukhal, A., Chen, Y., and Lu, L. "A note on the complexity of flow shop scheduling with transportation constraints", European Journal of $O p$ erational Research, 178, pp. 918-925 (2007).

33. Pan, J.C.H., Wu, C.L., Huang, H.C., and Su, C.S. "Coordinating scheduling with batch deliveries in a twomachine flow shop", International Journal of Advanced Manufacturing Technology, 40, pp. 607-616 (2009).

34. Soukhal, A., Oulamara, A., and Martineau, P. "Complexity of flow shop scheduling problems with transportation constraints", European Journal of Operational Research, 161, pp. 32-41 (2005).

35. Atashpaz, G.E. and Lucas, C. "Imperialist competitive algorithm: an algorithm for optimization inspired by imperialistic competition", IEEE Congress, pp. 46614667 (2007).

36. Seidgar, H., Kiani, M., Abedi, M., and Fazlollahtabar, H. "An efficient imperialist competitive algorithm for scheduling in the two-stage assembly flow shop problem", International Journal of Production Research, 52, pp. 1240-1256 (2014).

37. Rabiee, M., Zandieh, M., and Jafarian, A. "Scheduling of a no-wait two-machine flow shop with sequencedependent setup times and probable rework using robust meta-heuristics", International Journal of Production Research, 50, pp. 7428-7446 (2012).

38. Behnamian, J. and Zandieh. M. "A discrete colonial competitive algorithm for hybrid flowshop scheduling to minimize earliness and quadratic tardiness penalties", Expert Systems with Applications, 38, pp. 1449014498 (2011).
39. Shokrollahpour, E., Zandieh, M., and Dorri, B. "A novel imperialist competitive algorithm for bi-criteria scheduling of the assembly flowshop problem", International Journal of Production Research, 49, pp. 30873103 (2011).

40. Attar, S.F., Mohammadi, M., and TavakkoliMoghaddam, R. "A novel imperialist competitive algorithm to solve flexible flow shop scheduling problem in order to minimize maximum completion time", International Journal of Computer Applications, 28, pp. 27-32 (2011).

41. Karimi, N., Zandieh, M., and Najafi, A.A. "Group scheduling in flexible flow shops: a hybridised approach of imperialist competitive algorithm and electromagnetic-like mechanism", International Journal of Production Research, 49, pp. 4965-4977 (2011).

42. Javadian, N., Rezaeian, J., Khorshidian, H., and Rahmani, K. "Single machine preemptive scheduling by hybridized meta-heuristic approach", IEEE, pp. 750-753 (2011).

43. Carlier, J. "Ordonnancements a contraintes disjonctives", Recherche Perationelle/Operations Research, 12, pp. 333-351 (1978).

44. Reeves, C.R. "A genetic algorithm for flow shop sequencing", Computers \& Operations Research, 22, pp. 5-11 (1995).

45. Taillard, E. "Benchmarks for basic scheduling problems", European Journal of Operational Research, 64, pp. 278-285 (1993).

46. Montgomery, D.C., Design and Analysis of Experiments, 5th ed., Wiley, New York (2000).

47. Naderi, B., Zandieh, M., and Fatemi-Ghomi, S.M.T. "Scheduling job shop problems with sequence dependent setup times", International Journal of Production Research, 47, pp. 5959-5976 (2009).

48. Taguchi, G. "Introduction to quality engineering", White Plains, Asian Productivity Organization/UNIPUB (2000).

49. Qian, B., Wang, L., Hu, R., Wang, W.L., Huang, D.X., and Wang, X. "A hybrid differential evolution method for permutation flow-shop scheduling", International Journal of Advanced Manufacturing Technology, 38, pp. $757-777$ (2008).

50. Nawaz, M., Enscore, J.E., and Ham, I. "A heuristic algorithm for the m-machine, n-job flowshop sequencing problem", OMEGA, 11, pp. 91-95 (1983).

51. Mokhtari, H., Kamal-Abadi, I.N., and Cheraghalikhani, A. "A multi-objective flow shop scheduling with resource-dependent processing times: trade-off between makespan and cost of resources", International Journal of Production Research, 49(19), pp. 5851-5875 (2010).

52. Campbell, H.G., Dudek, R.A., and Smith, M.L. "A heuristic algorithm for the $\mathrm{n}$ job, $\mathrm{m}$ machine sequencing problem", Management Science, 16(10), pp. 630637 (1970). 


\section{Biographies}

Shahriar Mohammadi is a former Senior Lecturer at the University of Derby, UK. He also used to be a Network Consultant in the UK for more than fifteen years. He is currently a Lecturer in the Industrial Engineering Department of the University of K.N. Toosi, Iran. His main research interests and lectures are in the fields of networking, data security, network security, e-commerce and e-commerce security. He has published more than eighty papers in various journals and conferences as well as four books.

Ali Cheraghalikhani received his BSc degree from Iran University of Science and Technology, Tehran, Iran in 2007 and MS degrees from K.N. Toosi University of
Technology, Tehran, Iran in 2010, both in Industrial Engineering. Currently, he is a PhD candidate in the same field at K.N. Toosi University of Technology. His research background and interests mainly include supply chain management, algorithms and applied production and operations research.

Reza Ramezanian received $\mathrm{BSc}$, MSc, and $\mathrm{PhD}$ degrees, all in Industrial Engineering, from Iran University of Science and Technology, Tehran, Iran. He is currently an Assistant Professor of Industrial Engineering at K.N. Toosi University of Technology, Tehran, Iran and is reviewer of several international journals. His research interests include operation research, stochastic optimization, supply chain management, production planning, dynamic lot-sizing, and scheduling theory and applications. 